\title{
PRODUTIVIDADE CIENTÍFICA ACERCA DA NEOPLASIA MALIGNA BUCAL E DA REABILITAÇÃO BUCAL: UMA ANÁLISE BIBLIOMÉTRICA
}

\author{
Marta Regina Chaves Camilo Fernandes ${ }^{1}$, Carlos Roberto Lyra da Silva ${ }^{2}$, Jully Hannay Santos de Souza ${ }^{3}$, \\ Larissa Chaves Morais de Lima ${ }^{4}$, Isabel Cristina Ramos Vieira Santos ${ }^{5}$, Inácia Sátiro Xavier de França ${ }^{6}$
}

\begin{abstract}
RESUMO: Analisar resultados da recuperação de artigos sobre a temática neoplasia bucal e reabilitação bucal, publicados entre os anos de 1990 e abril de 2016, identificar a dispersão das publicações, examinar o padrão de autoria e citações na base de dados Scopus, a partir das Leis de Bradford e Lotka. Estudo bibliométrico, com abordagem descritiva e quantitativa. Realizada análise de artigos publicados em periódicos indexados na base de dados Scopus, utilizando-se os termos Mouth Neoplasms e Mouth Rehabilitation. Foram recuperados 28 artigos originais, publicados em 18 revistas, com distribuição irregular no período estudado. No momento da análise, identificou-se 222 citações com média de oito por documento. Medicina e odontologia se destacaram com publicações na temática. A Alemanha foi o país com maior produção científica. O estudo evidencia um quantitativo discreto de publicações e revela um campo que necessita do engajamento da enfermagem.
\end{abstract}

DESCRITORES: Neoplasia bucal; Reabilitação bucal; Enfermagem; Bibliometria.

\section{SCIENTIFIC PRODUCTIVITY IN RELATION TO MOUTH NEOPLASMS AND MOUTH REHABILITATION: A BIBLIOMETRIC ANALYSIS}

\begin{abstract}
The objectives of this study were to analyze the results of articles retrieved on the subject of mouth neoplasms and mouth rehabilitation, published between 1990 and April 2016, identify the dispersion of the publications, and examine authorship patterns and citations in the Scopus database, based on the Laws of Bradford and Lotka. This was a bibliometric study, with a descriptive and quantitative approach. An analysis of articles published in journals indexed in the Scopus database was performed, using the terms "Mouth Neoplasms" and "Mouth Rehabilitation". Twenty-eight original articles were retrieved, published in 18 journals, with irregular distribution in the period studied. At the time of analysis, 222 citations were identified with a mean of eight per document. The fields of medicine and dentistry stood out with publications on the theme. Germany had the largest scientific production. The study revealed a modest number of publications and the need for nursing engagement in this area of research.
\end{abstract}

DESCRIPTORS: Mouth neoplasms; Mouth rehabilitation; Nursing; Bibliometrics.

\section{PRODUCTIVIDAD CIENTÍFICA ACERCA DE LA NEOPLASIA MALIGNA BUCAL Y LA REHABILITACIÓN BUCAL: UN ANÁLISIS BIBLIOMÉTRICO}

RESUMEN: Analizar resultados de la recuperación de artículos sobre la temática neoplasia bucal y rehabilitación bucal, publicados entre los años 1990 y abril de 2016; identificar la dispersión de las publicaciones, examinar el estándar de autoría y citaciones en base de datos Scopus partiendo de las Leyes de Bradford y Lotka. Estudio bibliométrico, con abordaje descriptivo y cuantitativo. Se realizó análisis de artículos publicados en periódicos indexados en la base Scopus, utilizándose los términos Mouth Neoplasms y Mouth Rehabilitation. Pudieron recuperarse 28 artículos originales, publicados en 18 revistas, con distribución irregular en el período estudiado. Al momento del análisis de identificaron 222 citaciones, con promedio de ocho por documento. La Medicina y la Odontología se destacaron con publicaciones en la temática. Alemania fue el país de mayor producción científica. El estudio evidencia una cantidad discreta de publicaciones y revela un campo que necesita del compromiso de la enfermería.

DESCRIPTORES: Neoplasias de la Boca; Rehabilitación Bucal; Enfermería; Bibliometría.

${ }^{1}$ Enfermeira. Mestra em Enfermagem. Universidades de Pernambuco e Estadual da Paraíba. Recife, PE, Brasil.

${ }^{2}$ Enfermeiro. Pós-Doutorado em Enfermagem. Docente da Universidade Federal do Estado do Rio de Janeiro. Rio de Janeiro, RJ, Brasil.

${ }^{3}$ Discente de Enfermagem. Universidade de Pernambuco. Recife, PE, Brasil.

${ }^{4}$ Cirurgiã-Dentista. Mestranda em Odontologia. Universidade Estadual da Paraíba. Campina Grande, PB, Brasil.

${ }^{5}$ Enfermeira. Doutora em Saúde Pública. Docente de Pós-graduação em Enfermagem das Universidades de Pernambuco e Estadual da Paraíba. Recife, PE, Brasil.

${ }^{6}$ Enfermeira. Doutora em Enfermagem. Docente de Pós-graduação em Enfermagem das Universidades de Pernambuco e Estadual da Paraíba. Recife, PE, Brasil.

Autor Correspondente:

Marta Regina Chaves Camilo Fernandes

Universidade de Pernambuco

R. do Espinheiro, 690 - 52020-025 - Recife, PE, Brasil

E-mail: martfernands@gmail.com
Recebido: 04/01/2017

Finalizado: 15/09/2017 


\section{INTRODUÇÃO}

A neoplasia maligna constitui um problema de saúde pública mundial, dada a sua magnitude epidemiológica, social e econômica. De acordo com os dados da Agência Internacional para a Pesquisa em Câncer (IARC), da Organização Mundial da Saúde - OMS, a incidência no mundo cresceu $20 \%$ na última década ${ }^{(1)}$.

O câncer da cavidade oral é o décimo mais comum no mundo, dois terços dos quais é observado em países em desenvolvimento ${ }^{(1)}$. Essa doença ocupa, no biênio 2016/2017, o $5^{\circ}$ lugar na lista de incidência entre a população masculina brasileira, com aproximadamente 11.390 novos casos, e, entre as mulheres, a décima segunda colocação com 4.350 novos $\operatorname{casos}^{(2)}$.

Em muitos países, assim como no Brasil, os pacientes têm diagnóstico estabelecido na fase avançada ou metastática da doença, o que, somado à agressividade própria do tumor, dificulta o tratamento e piora significativamente o prognóstico ${ }^{(3)}$. O tratamento para esses pacientes habitualmente pode incluir a cirurgia, a radioterapia, a quimioterapia ou a combinação destes. Há, contudo, possibilidades de levar a inúmeros efeitos colaterais e secundários, como a deformidade bucal com sérios prejuízos nas funções mastigatória, deglutição e fonética, assim como nos relacionamentos interpessoais, tanto social quanto familiar ${ }^{(4-6)}$.

A reabilitação física e psicológica dos pacientes com tal deformidade, seu ajustamento familiar, social e ocupacional, dependem do trabalho de uma equipe multidisciplinar composta por enfermeiro, odontólogo, médico, nutricionista, fonoaudiólogo, terapeuta ocupacional, assistente social, fisioterapeuta, psicólogo e protético. A enfermagem, juntamente com os outros membros dessa equipe multiprofissional, desenvolve atividades que permitem a troca de conhecimentos, sistematizando informações e ações que se complementam, visando à reintegração desses pacientes à sociedade, através da comunicação oral, possibilitando assim uma melhor qualidade de vida ${ }^{(7)}$.

Em decorrência da importância atribuída à estimativa do câncer, é possível que estudiosos de diversas áreas do conhecimento tenham voltado suas pesquisas para focalizar novos procedimentos de cuidar de pessoas, visando à reabilitação de indivíduos acometidos pelo câncer da cavidade oral(5). Assim, conhecer a origem destas pesquisas, por meio de um estudo bibliométrico, poderá beneficiar os profissionais, em todos os níveis da assistência, bem como pesquisadores interessados pelo tema.

A bibliometria é uma ferramenta estatística que possibilita conhecer o quantitativo de periódicos nucleares que pressupõe ter os artigos mais relevantes publicados sobre uma determinada temática(8). Esta pesquisa está embasada pelas principais leis bibliométricas: Lei de Bradford (produtividade de periódicos) e Lei de Lotka (produtividade científica de autores) e, seguindo os princípios empíricos advindos da informação científica, permite mapear e gerar diversos indicadores de informação e produtividade, auxiliando a traçar o perfil da ciência e como conseguinte gerenciar estudos ${ }^{(9-10)}$.

Ao traçar o panorama sobre a produção científica na temática apresentada, este estudo permitirá estimar as possíveis lacunas e buscar a relevância de pesquisa, podendo contribuir para profissionais e pesquisadores da saúde ou de áreas correlatas, pois apresentará a distribuição da produção no tempo, área geográfica, área do conhecimento, o impacto dos periódicos e os autores mais produtivos.

Nesse sentido, os objetivos desta pesquisa foram analisar resultados da recuperação de artigos sobre a temática neoplasia bucal e reabilitação bucal, publicados entre os anos de 1990 e abril de 2016, identificar a dispersão das publicações, examinar o padrão de autoria e citações na base de dados Scopus, a partir das Leis de Bradford e Lotka.

\section{MÉTODO}

Trata-se de um estudo bibliométrico, do tipo descritivo e quantitativo que aborda a produção, disseminação e o uso da informação registrada eletronicamente em base de dados internacional.

A bibliometria tem sido aplicada em diversas áreas do conhecimento, como forma adequada de avaliar a produção científica e permitir a visualização da bibliografia de um determinado campo 
temático, visando à compreensão do assunto. Constitui uma ferramenta que permite explorar, mensurar e visualizar uma realidade multifacetada, operacionalizar o estudo da produção e uso da informação, bem como tratar, separar e classificar os dados registrados em fonte de informações ${ }^{(11)}$.

Dessa forma, a bibliometria promove a abertura do campo à inter e transdisciplinaridade, comportamento coerente com a contemporaneidade e a ação de promover a discussão entre os pares das instâncias de seus muros de domínio.

Esta pesquisa foi realizada respeitando as seguintes etapas: realização da busca, recuperação e preparação dos dados, tratamento bibliométrico utilizando as Leis de Bradford e Lotka, tratamento estatístico, representação gráfica e análise dos gráficos, além da exposição da interpretação dos dados.

O alicerce científico para o estudo se deu através da busca de termos contidos nos Descritores em Ciências da Saúde (DeCS) - "Neoplasia Bucal" e "Reabilitação Bucal". Em seguida, esses mesmos termos foram utilizados em inglês 'Mouth Neoplasms' e 'Mouth Rehabilitation' para recuperação de artigos na base de dados online da Scopus, que conta com um dos maiores bancos de dados utilizados pela comunidade científica internacional. A Scopus contém 50 milhões de registros, abrange mais de 21.000 periódicos revisados por pares e cerca de 5000 editores em todo o mundo ${ }^{(12)}$.

Foram utilizados os descritores associadamente, por meio dos recursos de pesquisa booleana AND ("Mouth Neoplasms" AND "Mouth Rehabilitation"). O idioma de escolha para a pesquisa foi o inglês por favorecer o intercâmbio global do conhecimento, podendo atingir um número maior de publicações e, portanto, um número maior de documentos recuperados.

A coleta de dados foi realizada em abril de 2016. Para os critérios de inclusão - artigos originais, disponibilizados de forma gratuita e na íntegra, na internet, que tivessem os termos utilizados no título, no corpo do resumo ou nos descritores, não havendo limitações para qualquer subárea. Foram recuperados artigos publicados no período de janeiro de 1990 até abril de 2016. Os critérios de exclusão foram: qualquer documento que representasse orientações para procedimentos ou diretrizes de sociedade de especialistas (guideline).

Os dados recuperados na Scopus foram tratados à luz da estatística descritiva, com apresentação em forma de distribuição de frequência simples e relativa. A mensuração do SCImago Journal RankSJR, foi retirado diretamente da página da SCImago, também no mesmo período da recuperação dos documentos.

Utilizou-se a métrica alternativa de impacto de periódicos, a qual se fundamenta na ideia de que cada citação é criada de forma distinta. Com o SJR, a área de pesquisa, a qualidade e a reputação do periódico tem um impacto direto sobre o valor de uma citação. Uma citação de uma fonte com um SJR relativamente alto vale mais do que uma citação de uma fonte com um SJR menor.

Além disso, foram aplicadas a Lei de Bradford ou Lei da Dispersão que possibilita estimar o grau de relevância de periódicos que atuam em áreas do conhecimento específicas, e a Lei de Lotka, a qual analisa a produtividade científica dos autores, ou seja, verifica a contribuição de cada um para o desenvolvimento científico em sua área de conhecimento ${ }^{(9)}$.

\section{- RESULTADOS}

Foram recuperados 28 documentos, classificados como artigos originais. Não houve necessidade de excluir nenhum artigo, pois todos contemplavam os critérios de inclusão. Para o período delimitado na pesquisa de 1990 a abril de 2016, foram encontrados, como mostra a Tabela 1, artigos com distribuição irregular em anos de publicação. No espaço temporal descrito, os anos que se destacam com maior número de documentos são 1992, 1999 e 2009. 
Tabela 1 - Total de artigos recuperados por ano de publicação. Recife, PE, Brasil, 2016

\begin{tabular}{cc} 
Ano & Total de Artigos \\
\hline 2013 & 1 \\
\hline 2012 & 1 \\
\hline 2011 & 1 \\
\hline 2009 & 4 \\
\hline 2008 & 1 \\
\hline 2007 & 1 \\
\hline 2006 & 1 \\
\hline 2005 & 1 \\
\hline 2004 & 1 \\
\hline 1999 & 5 \\
\hline 1997 & 1 \\
\hline 1996 & 1 \\
\hline 1993 & 3 \\
\hline 1992 & 5 \\
\hline 1990 & 1
\end{tabular}

Fonte: Base de dados Scopus (2016)

Quanto à temática apresentada, constatou-se que dos 28 artigos, $12(42,8 \%)$ tratavam da reabilitação, sete (25\%) eram estudos epidemiológicos, seis $(21,4 \%)$ sobre técnicas cirúrgicas, dois $(7,1 \%)$ referiamse a atitude profissional e um $(3,5 \%)$, sobre cuidados pré e pós-radioterapia.

Com relação às áreas de interesse descritas na base de dados Scopus, verificadas em 2016 e relacionadas aos artigos recuperados de acordo com o refinamento da busca de dados, notou-se que a maior frequência era de interesse exclusivo da Medicina com 12 (42,8\%), seguido da Odontologia com cinco $(17,8 \%)$ e por aqueles de interesse mútuo que corresponde às áreas de Medicina e Odontologia com $10(35,7 \%)$, acrescido também aquele de área indefinida com uma (3,5\%).

Os 28 documentos recuperados foram publicados em 18 periódicos revisados por pares. O SCImago Journal \& Country Rank (SJCR) é um portal disponível publicamente que inclui os periódicos e indicadores científicos dos países desenvolvidos a partir das informações contidas no banco de dados Scopus ${ }^{\circledast}$ (Elsevier BV). Esta plataforma tem o seu nome a partir do indicador de SCImago Journal Rank (SJR) que mostra a visibilidade dos periódicos desde $1996^{(13)}$.

Para a mensuração do Impacto por Publicação (IPP) e SJR, foram considerados os 10 primeiros periódicos cujos resultados variaram para o IPP, de 0,47 a 2,67 com média de 1,94 e para o SJR, de 0,22 a 1,99 com média de 0,87. Para os periódicos que se destacaram nas respectivas métricas, foram identificados: Plastic and Reconstructive Surgery com SJR (2015) 1,99 e IPP (2015) 2,67 e, Periodontology 2000 com SJR (2015) 1,84 e IPP (2015) 4,79.

A Tabela 2, conforme prevê a Lei de Bradford, mostra a ordenação dos periódicos segundo zonas de produtividade e indica que os dados empíricos dos periódicos e artigos não estão em posição de linha de produtividade, de acordo com os cálculos teóricos. Nesta pesquisa foram consideradas três zonas de produtividade para os artigos recuperados no estudo. É importante mencionar os periódicos que se destacaram em produtividade na temática, durante o espaço temporal descrito na pesquisa. $\mathrm{O}$ periódico Mund-Kiefer-und Gesichtschirurgie encontra-se na zona 1, com seis artigos publicados; os periódicos Fortschrtte der Kiefer-und Gesichtschirurgie e Laringo-Rhino Otologie estão localizados na zona 2, respectivamente com cinco e dois artigos publicados. Os demais periódicos, 15, apresentaram apenas um artigo publicado. 
Tabela 2 - Tabela Clássica de Bradford. Recife, PE, Brasil, 2016

\begin{tabular}{ccccccc} 
Zonas & $\begin{array}{c}\text { Cálculo Teórico } \\
\text { Total de Artigos }\end{array}$ & $\mathbf{N}^{\mathbf{0}}$ periódico & $\begin{array}{c}\text { Cálculo Empírico } \\
\text { Total de Artigos }\end{array}$ & $\mathbf{N}^{\mathbf{0}}$ periódico & mB & Em linha \\
\hline 1 & 9 & 2 & 6 & 1 & & Não \\
\hline 2 & 9 & 4 & 7 & 2 & 2 & Não \\
\hline 3 & 10 & 12 & 15 & 15 & 7,5 & Não
\end{tabular}

Fonte: dados da pesquisa

$\mathrm{mB}=$ multiplicador de Bradford

A Tabela 3 mostra que o número de periódicos em cada zona aumenta na medida em que a produtividade diminui, evidenciando rentabilidade decrescente entre as três zonas identificadas.

Tabela 3 - Divisão de Periódicos por zona de produtividade. Recife, PE, Brasil, 2016

\begin{tabular}{|c|c|c|c|c|c|c|c|c|c|}
\hline $\mathbf{Z}$ & A & $\Sigma \mathbf{A}$ & $\%$ A & $\% \Sigma A$ & $\mathbf{P}$ & $\Sigma P$ & $\% \mathrm{P}$ & $\% \Sigma \mathrm{P}$ & $\mathrm{mB}$ \\
\hline $1^{a}$ & 6 & 6 & 21,4 & 21,4 & 1 & 1 & 5,5 & 5,5 & -- \\
\hline $2^{a}$ & 7 & 13 & 25 & 46,4 & 2 & 3 & 11,1 & 13,3 & 2 \\
\hline $3^{a}$ & 15 & 28 & 53,5 & 100 & 15 & 18 & 83,3 & 100 & 7,5 \\
\hline
\end{tabular}

$\mathrm{Z}=$ zonas de produtividade; $\mathrm{A}=$ artigos; $\Sigma \mathrm{A}=$ somatório dos artigos; $\% \mathrm{~A}=$ percentual dos artigos; \% $\Sigma \mathrm{A}=$ percentual do somatório dos artigos; $\mathrm{P}=$ periódicos; $\Sigma \mathrm{P}=$ somatório dos periódicos; \% $\mathrm{P}=$ percentual dos periódicos; \% $\% \mathrm{P}=$ percentual do somatório dos periódicos; $\mathrm{mB}=$ multiplicador de Bradford; $\mathrm{XmB}=$ Valor médio do multiplicador de Bradford.

Fonte: Dados da pesquisa

A divisão de periódicos por zona de produtividade exposta na Tabela 3 mostra que a primeira zona equivale a 5,5\% dos periódicos da distribuição com $21,4 \%$ do total de artigos publicados, evidenciando um núcleo de produção na temática em estudo, e a última zona apresenta significativa dispersão da literatura científica, concentrando 83,3\% de periódicos com 53,5\% de artigos publicados.

No que tange aos valores do mB (multiplicador de Bradford), resultado da divisão do número de periódicos de uma zona pela anterior, oscilam significativamente entre uma zona e outra. No entanto, o valor do XmB (valor médio do multiplicador de Bradford) distancia dos valores individuais do $\mathrm{mB}$ das zonas 2 e 3, confirmando a diferença de produtividade entre os periódicos.

Os documentos recuperados foram publicados em diferentes países, com destaque para Alemanha $(n=5)$, Reino Unido ( $n=3)$ e Estados Unidos da América $(n=2)$, conforme apresentado na Tabela 4.

Tabela 4 - Países com artigos publicados. Recife, PE, Brasil, 2016

\begin{tabular}{llc} 
Ordem* & País & Artigos publicados \\
\hline $1^{\circ}$ & Alemanha & 5 \\
\hline $2^{\circ}$ & Reino Unido & 3 \\
\hline $3^{\circ}$ & Estados Unidos da América & 2 \\
\hline $4^{\circ}$ & Áustria & 1 \\
\hline $4^{\circ}$ & França & 1 \\
\hline $4^{\circ}$ & Itália & 1 \\
\hline $4^{\circ}$ & Holanda & 1
\end{tabular}

Fonte: Base de dados Scopus (2016)

* Diferentes países tiveram o mesmo número de ordem por conterem o mesmo número de artigos publicados. 
A Tabela 5 evidencia os dez autores com maior visibilidade em citações no período da análise dos dados, e quantifica, através do índice- $\mathrm{H}$, a produtividade e o impacto desses autores baseando-se nos seus artigos mais citados. Para esta avaliação foi selecionado o primeiro autor de cada um dos 28 artigos analisados e extraídos os 10 autores com maior destaque em número de citações no espaço temporal determinado na pesquisa.

Tabela 5 - Top 10 de autoria direta com maior número de citações. Recife, PE, Brasil, 2016

\begin{tabular}{llccc}
\hline Ordem* & Autor & $\mathbf{N}^{\mathbf{0}}$ de Artigos & $\mathbf{N}^{\mathbf{0}}$ de Citações & índice-H \\
\hline $1^{\circ}$ & Nicoletti G. & 1 & 46 & 5 \\
\hline $2^{\circ}$ & Chan MFWY. & 1 & 41 & 8 \\
\hline $3^{\circ}$ & Pace-Balzan A. & 3 & 32 & 6 \\
\hline $4^{\circ}$ & Kreeft A. & 1 & 20 & 5 \\
\hline $5^{\circ}$ & Grötz Knut A. & 1 & 15 & 19 \\
\hline $6^{\circ}$ & Bootz F. & 1 & 14 & 22 \\
\hline $7^{\circ}$ & Alani A. & 1 & 10 & 9 \\
\hline $8^{\circ}$ & Schmelzeisen R. & 1 & 8 & 37 \\
\hline $8^{\circ}$ & Méningaud JP. & 1 & 8 & 18 \\
\hline $9^{\circ}$ & Betz T. & 1 & 7 & 5
\end{tabular}

*Diferentes autores tiveram o mesmo número de ordem por conterem o mesmo número de citações e artigos publicados. Fonte: Base de dados Scopus (2016)

É importante mencionar os três artigos com maior visibilidade em citações e suas respectivas autorias diretas: 1. Chewing and swallowing after surgical treatment for oral cancer: Functional evaluation in 196 selected cases, autoria direta de Nicoletti G., com 46 citações; 2. Oral Rehabilitation with ImplantRetained Prostheses Following Ablative Surgery and Reconstruction with Free Flaps, autoria direta de Chan MFWY., com 41 citações; 3. The surgical dilemma of 'functional inoperability' in oral and oropharyngeal cancer: Current consensus on operability with regard to functional results, autoria direta de Kreeft A., com 20 citações.

\section{DISCUSSÃO}

O primeiro artigo disponível indexado na base de dados da Scopus data de 1990 e o último em 2013, o que corresponde a um espaço temporal de 23 anos. Observou-se um aumento de publicações nos anos de 1992, 1993, 1999 e 2009, no entanto esse crescimento não foi sustentado nos anos seguintes.

Os periódicos foram distribuídos em zonas de produtividade, de modo que essas apresentaram frequências absolutas de artigos com valores distintos. A primeira zona forma o núcleo de produtividade, contendo o periódico que mais publica, supostamente de maior qualidade ou relevância para área de conhecimento. A última zona é considerada como faixa de dispersão, contendo os periódicos de produção inferior.

Os dados empíricos não estão em linha com a teoria de Bradford, visto que o comportamento da distribuição de periódicos entre as zonas não foram compatíveis. Pode-se inferir que a quantidade de artigos é muito irrelevante quando comparada com a quantidade de periódicos. O multiplicador de Bradford (mB) confirma a dispersão contida na Tabela 2.

A média de publicação foi de 1,12 artigo/ano. Apesar da grande dispersão evidenciada, é possível afirmar que o tema ainda não alcançou sua maturidade, muito menos se encontra obsoleto. Este fato pode ser constatado quando a Agência Internacional de Pesquisa para o Câncer (IARC) ${ }^{(1)}$ apresenta dados com tendência ascendente para todos os tipos de câncer, na população mundial, nos próximos 14 anos, e que no Brasil a estimativa de casos novos, especificamente para os de cavidade oral, em 2016 
foi de $15.490^{(2)}$. Desta forma, o estudo possibilita o desenvolvimento de pesquisas futuras de forma comparativa entre o que foi coletado no cenário internacional e a forma como o teor do estudo é discutido no Brasil.

Sobre a temática câncer de cavidade oral, percebe-se que a maior frequência dos artigos analisados deu ênfase à reabilitação, e apenas um, ao longo de 23 anos de publicações, de autoria médica, abordou os cuidados pré e pós-radioterapia ${ }^{(14)}$. Embora a preocupação com a reabilitação desses pacientes tenha se manifestado pela frequência de artigos publicados, os resultados chamam a atenção tanto pelo inexpressivo número de estudos sobre os cuidados necessários para eles, quanto pela ausência de publicação pela enfermagem.

Vale lembrar a definição de enfermagem, formulada pela Associação Americana de Enfermagem ${ }^{(15)}$, na qual está incluída a proteção, promoção e otimização da saúde e habilidades, o alívio do sofrimento através do diagnóstico e tratamento da resposta humana e o cuidado apropriado ao indivíduo, este último alicerçado nas melhores evidências científicas. Deste modo, os resultados apontam para a necessidade de estudos que revelem a necessária inclusão da enfermagem nos cuidados ao paciente com câncer da cavidade oral.

No cenário internacional, é importante mencionar que a Alemanha é o país que se destaca em produção científica na base de dados da Scopus com artigos neste tema. Talvez fundamentado pela taxa de incidência deste tipo de câncer $(29,5)$ comparado aos dois outros países que seguem na relação do maior numero de artigos publicados, ou seja, Reino Unido no qual a taxa registrada em 2012 foi de 19,9 e Estados Unidos que apresenta uma taxa para o ano de 2014 de 10,5 $5^{(16-17)}$.

Nesse estudo, foi possível estimar maior visibilidade dos periódicos Plastic and Reconstructive Surgery e Periodontology 2000, ambos com SJR >1, supostamente com maior qualidade ou relevância para a temática em estudo, na base de dados selecionada. O SJR identifica que a qualidade e a reputação dos periódicos têm um efeito direto sobre o valor de uma citação. Contudo, o aspecto qualitativo dos periódicos a partir do SJR evidenciou a distância que os artigos publicados estão dos periódicos de alto impacto porque a maioria dos periódicos analisados apresentou SJR inferior a 1.

$\mathrm{Na}$ relação autoria direta, considerando os 10 autores mais citados, foi observado o fenômeno conhecido como processo elitista ou Efeito Mateus na Ciência, isto é, "aos que mais têm será dado em abundância e, aos que menos têm, até o que têm Ihes será tirado"(18:3). Sob essa perspectiva, podese concluir que os quatro primeiro autores, supostamente de maior prestígio, produzem muito e consequentemente são mais citados, como mostram seus índices-H. Quanto mais se publica, mais parece que se facilita publicar um novo trabalho e os pesquisadores que publicam resultados mais interessantes ganham mais reconhecimento e acesso a recursos para melhorar sua pesquisa ${ }^{(19)}$. Inversamente, um maior número de autores, supostamente de menor prestígio, apresentou pouca produtividade.

A divulgação científica na área da saúde ocorre, principalmente, sob o formato de artigo, creditando, portanto, a esse tipo de veículo uma das principais formas de comunicação no meio acadêmico. Portanto, é necessário que os periódicos sejam, com certa frequência, analisados, contribuindo para o aprimoramento dos artigos publicados, buscando tratar da educação continuada e do rigor da produção científica ${ }^{(20)}$.

\section{- CONSIDERAÇÕES FINAIS}

Por meio da mensuração da produtividade científica, através da bibliometria, foi possível estimar a magnitude na temática neoplasia bucal e reabilitação bucal, que neste campo do conhecimento, a área da enfermagem não apresentou qualquer produção registrada na base de dados Scopus. A maior frequência foi de interesse exclusivo da Medicina, com enfoque na reabilitação.

O estudo mostra que o tema ainda não atingiu sua maturação científica do ponto de vista da quantidade de produção, sobretudo pela ausência de periódicos específicos na área de enfermagem. Ao que tudo indica, talvez o tema, ainda não despertado na enfermagem, uma possibilidade concreta de investigação capaz de contribuir para qualidade de vida de pacientes oncológicos necessitados de 
reabilitação oral, em que pese o fato de que a sua especificidade esteja direcionada para a medicina e odontologia. Contudo, para os resultados apresentados, há que se considerar a limitação do estudo, relacionada ao uso de uma única base de dados.

\section{REFERÊNCIAS}

1. World Health Organization (WHO). Internarnational Agency for Research on Cancer (IARC). Section of câncer information. [Internet] 2013 [acesso 22 jun 2015]. Disponível: https://www.iarc.fr/.

2. Instituto Nacional de Câncer (INCA). José Alencar Gomes da Silva. Coordenação de Prevenção e Vigilância. Estimativa 2016: incidência de Câncer no Brasil. Rio de Janeiro: INCA; 2015.

3. Nemotto RP, Victorino AA, Pessoa GB, da Cunha LLG, da Silva JAR, Kanda JL, et al. Oral câncer preventive campaigns: are we reaching the real target?. Braz J Otorhinolaryngol. [Internet] 2015;81(1) [acesso em 22 jun 2015]. Disponível: http://dx.doi.org/10.1016/j.bjorl.2014.03.002.

4. Thompson CF, St John MA, Lawson G, Grogan T, Elashoff D, Mendelsohn AH. Diagnostic value of sentinel lymph node biopsy in head and neck cancer: a metaanalysis. Eur Arch Otorhinolaryngol. [Internet] 2013;270(7) [acesso em 31 ago 2015]. Disponível: http://dx.doi.org/10.1007/s00405-012-2320-0.

5. de Oliveira JMB, Pinto LO, Lima NGM, de Almeida GCM. Percepção dos Acadêmicos sobre o Câncer de Boca. Rev. bras. cancerol. [Internet] 2013;59(2) [acesso em 31 ago 2015]. Disponível: http://www.inca.gov.br/rbc/n_59/ $\mathrm{v} 02 / \mathrm{pdf} / 08$-cancer-de-boca-avaliacao-do-conhecimento-de-academicos-de-odontologia-e-enfermagemquanto-aos-fatores-de-risco-e-procedimentos-de-diagnostico.pdf.

6. Eadie TL, Lamvik K, Baylor CR, Yorkston KM, Kim J, Amtmann D. Communicative participation and quality of life in head and neck cancer. Ann Otol Rhinol Laryngol. [Internet] 2014;123(4) [acesso em 31 ago 2015]. Disponível: http://dx.doi.org/10.1177/0003489414525020.

7. de Menezes MFB, Camargo TC, Guedes, MTS, de Alcântara LFFL. Câncer, pobreza e desenvolvimento humano: desafios para a assistência de enfermagem em oncologia. Rev. Latino-Am. Enfermagem. [Internet] 2007;15(n.esp) [acesso em 12 dez 2015]. Disponível: http://dx.doi.org/10.1590/S0104-11692007000700011.

8. da Cunha MV. Os periódicos em ciência da informação: uma análise bibliométrica. Ci. Inf. 1985;14(1):37-45.

9. Araújo CAA. Bibliometria: evolução histórica e questões atuais. Em Questão. [Internet] 2006;12(1) [acesso em 31 ago 2015]. Disponível: http://seer.ufrgs.br/index.php/EmQuestao/article/view/16.

10. Guedes VLS, Borschiver S. Bibliometria: uma ferramenta estatística para a gestão da informação e do conhecimento, em sistemas de informação, de comunicação e de avaliação científica e tecnológica. CINFORMEncontro Nacional de Ciência da Informação; 2005.

11. Sarcado MS. Publicação científica derivada das dissertações e teses na interface entre educação física e educação especial [dissertação]. São Carlos (SP): Universidade Federal de São Carlos; 2006.

12. Scopus: SciVerse Scopus fact sheet. SciVerse ${ }^{\circledR}$ Scopus. [Internet] 2013 [acesso em 14 set 2016]. Disponível: http://www.elsevier.com/online-tools/scopus.

13. SCImago. SJR - SCImago Journal \& Country Rank. [Internet] 2015 [acesso em 21 jul 2016]. Disponível: http:// www.scimagojr.com.

14. Borowski B, Margainaud JP. Orodental care before and after radiotherapy affecting the oral cavity. Rev Odontostomatol (Paris). 1990;19(2):151-5.

15. American Nurses Association (ANA). Nursing scope and standards of practice. $3^{\mathrm{a}}$ ed. Silver Spring: ANA; 2015.

16. Ferlay J, Steliarova-Foucher E, Lortet-Tieulent J, Rosso S, Coebergh JWW, Comber H, et al. Cancer incidence and mortality patterns in Europe: Estimates for 40 countries in 2012. Eur J Cancer. 2013;49(6):1374-403.

17. National Institute of dental and craniofacial research (NIH). Oral cancer incidence (new cases) by age, race and gender. [Internet] 2016 [acesso em 25 nov 2016]. Disponível: http://www.nidcr.nih.gov/DataStatistics/ 
FindDataByTopic/OralCancer/OralCancerIncidence.htm.

18. Merton RK. The Mathew effect in science. Science. [Internet] 1968;159(3810) [acesso em 01 set 2016 ]. Disponível: http://dx.doi.org/10.1126/science.159.3810.56.

19. Maltrás B. Los indicadores bibliométricos: fundamentos y aplicación al análisis de la ciencia. Gijón: Trea; 2003.

20. de Souza-Silva JR, Pires IH, Blascovi-Assis SM, de Paula CS. Análise da produção científica de dez anos da revista Psicologia: Teoria e Prática. Psicol. teor. prat. [Internet] 2010;12(3) [acesso em 17 dez 2016]. Disponível: http://pepsic.bvsalud.org/scielo.php?script=sci_arttext\&pid=S1516-36872010000300013. 